\title{
Childhood tuberculosis in an urban population in South Africa: burden and risk factor
}

\author{
A van Rie, N Beyers, R P Gie, M Kunneke, L Zietsman, P R Donald
}

\begin{abstract}
Aim-To study the epidemiology of childhood tuberculosis (TB) in a developing country.

Setting-Two urban communities of Cape Town, South Africa with a TB case notification rate of $1149 / 100000$.

Design-Retrospective descriptive study using the national population census (1991), 10 year official TB notification records, and a geographical information system.

Results-The case notification rate of TB in children 0-5 years old was 3588 cases/ 100000 children aged $0-5$ years, 3.5 times the case notification rate in adults. Children (0-14 years) accounted for $39 \%$ of the total case load. Childhood TB case notification rate correlated with parental education $(r=-0.64)$, annual household income $(r=-0.6)$ and crowding $(r=0.32)$.

Conclusion-Children, especially those living in poor socioeconomic conditions, form an important epidemiological group and account for a notable proportion of the morbidity caused by TB. Efforts to improve TB control must therefore not only target adults (case detection and cure of infectious cases) but also children (screening of child contacts of adult cases) and the socioeconomic living conditions. (Arch Dis Child 1999;80:433-437)
\end{abstract}

Keywords: tuberculosis; risk factors; developing world

Interest in tuberculosis (TB) has been reawakened worldwide by its global resurgence since the mid-1980s. The largest burden of TB lies in low income countries. However, the only data on the increase of childhood TB have been reported in the US, where there was a $39 \%$ increase in cases between 1987 and $1990 .{ }^{1}$ Nevertheless, the interest in childhood TB remains limited. Childhood $\mathrm{TB}$ is often regarded as unimportant in the epidemiology of TB because $>95 \%$ of children with TB are sputum smear negative and therefore do not contribute to the immediate spread of the disease. Childhood TB, however, is a marker for ongoing transmission of infection within a community and infected children represent the pool from which a large proportion of future cases of adult TB will arise. ${ }^{2}$

Furthermore, TB is an important cause of morbidity and mortality in children. The World Health Organisation estimated that 1.3 million cases of TB and 450000 deaths from TB occur annually among children in develop- ing countries. ${ }^{4}$ Historically, the highest mortality from TB ever reported was in children aged $0-4$ years in the middle of the 19 th century in Wales-600 deaths/100 000 children. ${ }^{5}$ The youngest children carry the biggest burden for three main reasons: firstly, they are more prone to develop severe extrapulmonary TB disease (such as meningitis and miliary TB); secondly, they tend to develop severe pulmonary disease with bronchial obstruction; and thirdly, young children are more likely to develop disease after being infected. The risk of developing disease after infection with $\mathrm{Myco-}$ bacterium tuberculosis has been estimated to be $5-10 \%$ in adults, $15 \%$ in adolescents, $24 \%$ in children $1-5$ years old, and $43 \%$ in infants $<1$ year old.

It is difficult to establish an exact picture of the incidence of TB in children. This is because the diagnosis is much less accurate than in adults and depends on a combination of clinical features (including tuberculin skin test and chest radiography) and contact history with an adult index case. ${ }^{5}$ More than $50 \%$ of childhood TB cases are asymptomatic and thus the majority will not be diagnosed in developing countries because of the lack of radiographic facilities. Therefore, the International Union Against Tuberculosis and Lung Disease has stated that reliable information on the incidence of TB in childhood can be obtained only in developed countries. ${ }^{5}$

For this study we selected an area with a population living in conditions typical of a developing country, with one of the highest reported TB case notification rates in the world but with access to a relatively sophisticated medical infrastructure.

The aims of the study were to gain data on the burden of childhood TB in a community of a developing country and to examine the risk factors of childhood TB in such a community.

\section{Methods}

The study area consists of two communities of Cape Town, South Africa with a total surface area of $2.42 \mathrm{~km}^{2}$ and a population of 34294 , of which $99.7 \%$ are coloured. A primary health care clinic and an adjacent tertiary hospital serve the area. The TB case notification rate in these two suburbs is reported as more than $1000 / 100000,{ }^{8}$ the birth rate is $29.3 / 1000$ population, and the infant mortality rate is 38/1000 live births. During the study period (1985 to 1994 ) the HIV prevalence was low. In 1991 , only $0.37 \%$ of pregnant women living in the Western Cape province who were screened anonymously were seropositive for HIV. ${ }^{9}$ The national TB programme during the study 


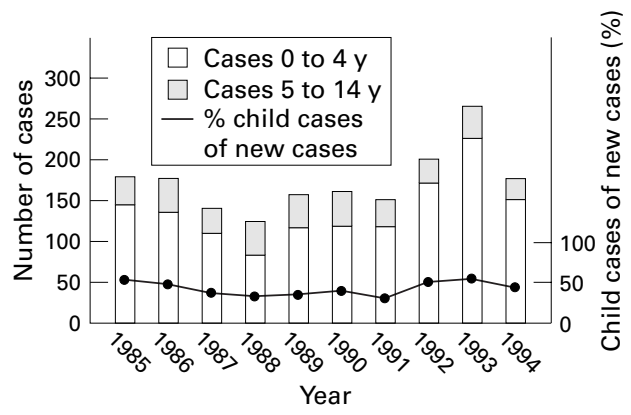

Figure 1 Age distribution of childhood TB cases. The bar graph shows the annual childhood case load in absolute numbers for the 10 year period of the study. The line represents the annual childhood case load as a percentage of all notified new cases.

period required the notification of all new cases of active TB and treatment (six months' isoniazid, rifampicin, and pyrazinamide) under supervision at the local health authority clinic. All adult household members and children under 5 years in contact with an index case were requested to visit the clinic to be screened for TB. Since 1972 BCG vaccination in the neonatal period was compulsory and the BCG coverage in the area is greater than $97 \%$.

Ten year official notification records (1985 to 1994) provided the name, address, age, and type of TB of each notified case. All duplicate notifications, previously treated patients, and children reported as "tuberculin skin test positive only" were eliminated to avoid the inaccuracies of a notification system. ${ }^{10}$ This allowed us to create a dataset consisting of only new cases. The home addresses of these individual patients were geographically mapped by using a computer based geographical information system.

The 1991 population census data divide the two suburbs into 39 enumerator subdistricts (ESD) with an average population of 900 people (minimum 627 and maximum 1267). The census report provided us for each ESD with demographic variables such as age and sex, social variables such as number of households, education, and type of housing, and economic variables such as employment and annual income per capita. From this data, we calculated one value for each ESD for crowding, economic status, and maternal and paternal education level. Crowding was calculated as

Table 1 Age distribution, sex ratio, and type of $T B$ in children in South Africa and the US

\begin{tabular}{lcl}
\hline & $\begin{array}{l}\text { South Africa } \\
1985-94\end{array}$ & $\begin{array}{l}\text { US (Alabama) } \\
\text { 1983-93 }\end{array}$ \\
\hline Child cases as \% of new cases & \\
0-15 years & 46 & $2-5$ \\
0-5 years & 36.5 & \\
6-15 years & 9.5 & \\
Sex ratio (male/female) & 0.98 & 0.94 \\
Age distribution (\%) & & \\
0-1 year & 37 & 41 \\
2-4 years & 42 & 22 \\
5-9 years & 17 & 16 \\
10-14 years & 4 & 21 \\
Type of TB (\%) & & \\
Pulmonary & 98 & 82.5 \\
Extrapulmonary & 2 & 17.5 \\
\hline
\end{tabular}

The first column gives the results of this study, the second column shows the results of a study conducted by Kimerling et al. ${ }^{11}$ the average number of adults ( $>15$ years of age) per room (sleeping and living rooms). Economic status was calculated as the average annual household income. Parental education level was calculated as the percentage of female and male adults ( $>15$ years of age) who had completed primary school education.

The TB notification data of 1991 were linked with the 1991 ESD data using the geographical information system. The TB case notification rate for each ESD was calculated using the notifications and the population in each ESD. Age specific TB case notification rates were calculated by using the notifications of a specific age group as the numerator and the age specific population as denominator. The TB case notification rates were correlated with crowding, economic status, and maternal and paternal education on the level of each ESD.

Correlations were analysed using Spearman rank correlation coefficients.

\section{Results}

TRENDS OVER TIME: STUDY PERIOD 1985 TO 1994 Case load

The total annual case load during the 10 year period varied from 327 to 458 and the annual childhood case load from 125 to 268 . The percentage of childhood cases as percentage of the total case load ranged from $37 \%$ to $58 \%$ (fig 1 ). Among all childhood cases, the proportion of children 0-4 years old ranged from $25 \%$ to $49 \%$ and the proportion of children $5-14$ years old ranged from $7 \%$ to $12 \%$. The case load showed no steady increase or decrease during the 10 year period.

\section{Childhood morbidity and mortality}

From 1985 to 1994 , there were 1383 cases of TB in children $0-4$ years old and 361 cases in children 5-14 years old, representing $36 \%$ and $9 \%$ of the total case load, respectively (table 1). ${ }^{11}$ Of the 1744 children with TB, $60(3.4 \%)$ were younger than 1 year old, $587(33.6 \%)$ were 1 year old, $354(20.3 \%) 2$ years old, 216 $(12.4 \%) 3$ years old, $166(9.5 \%) 4$ years old, $293(16.8 \%) 5-9$ years old, and $68(3.9 \%)$ 10-14 years old. The number of male and female children suffering from TB was similar.

Of the 1744 children with TB, 1710 (98\%) were notified as pulmonary TB (primary complex, hilar adenopathy, progressive primary disease), and $34(2 \%)$ as extrapulmonary TB (9 tuberculous meningitis, 10 pleural effusion, 5 bone, 4 lymphadenopathy, 1 genitourinary, 1 abdominal, and 4 others). One child died of tuberculous meningitis.

STUDY YEAR: 1991

Age specific $T B$ case notification rate

The differences in TB case notification rate between the different age groups was striking (fig 2). The case notification rate of TB in the total population of the two suburbs was 1149 / 100000 population. In adults ( $>15$ years old) the case notification rate was $1029 / 100000$ adults, in children $6-14$ years old the case notification rate was 332/100 000 children aged 6-14 years, and in children up to 5 years old 
A

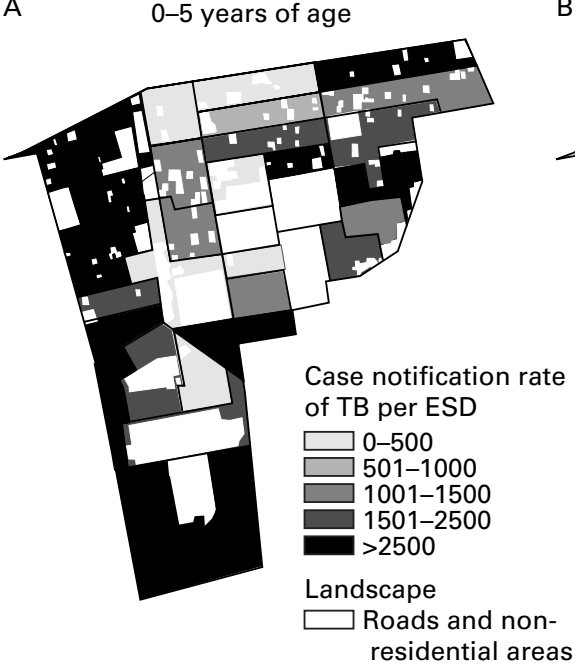

B

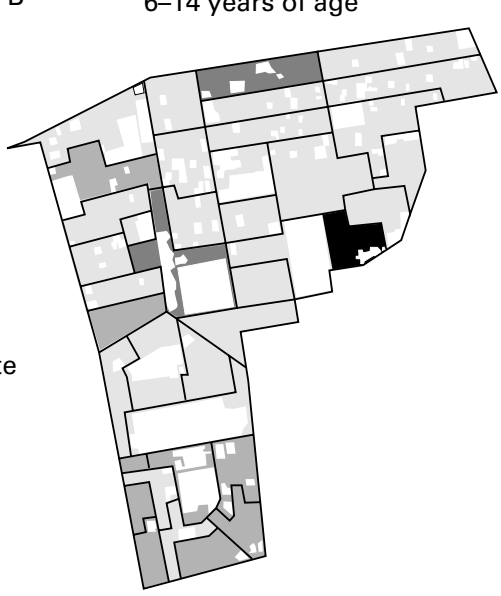

C $\quad>15$ years of age

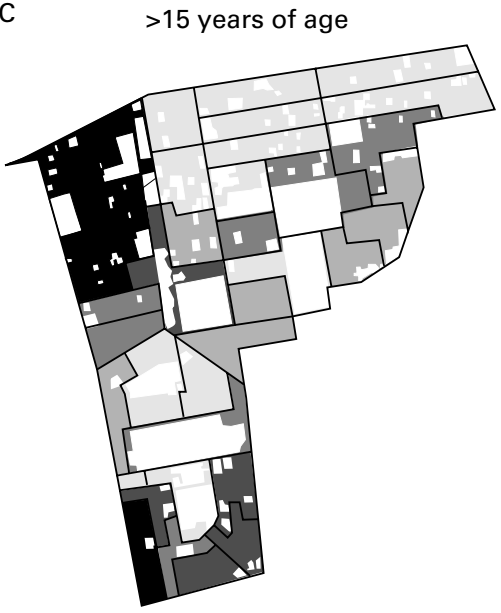

$250 \quad 0 \quad 2505007501000$

$\mathrm{m}$

Figure 2 Age specific TB case notification rates and their geographical distribution within two communities. The age specific case notification rate of TB is shown for: $(A)$ children 0-5 years old: $(B)$ children 6-14 years old; and $(C)$ adults. The geographical variation of the TB case notification rate for each age group is shown by mapping the age specific TB case notification rate for each of the 39 ESDs.

the case notification rate was 388/100 000 children aged $0-5$ years. Apart from the influence of age on the case notification rate of TB, there was a major geographical variation of the TB case notification rate within the community for each age group. The TB case notification rate in the different ESDs ranged from 0 to $14062 / 100000$ in children $0-5$ years old, from 0 to $3333 / 100000$ in children $6-14$ years old, and from 0 to $2847 / 100000$ in adults.

Social and economic risk factors for childhood $T B$ All the traditional risk factors investigated (crowding, economic status, and education) were significantly related with the TB case notification rate in children $0-5$ years old. Parental education $(r=-0.60, \mathrm{p}=0.000046)$ and annual household income $(r=-0.64$, $p=0.000015)$ showed the strongest correlation with the case notification rate of childhood TB (fig 3). The weakest correlation was between crowding and TB case notification rate in young children $(r=0.32, \mathrm{p}=0.046)$. In the study area the median annual household income in 1991 was 22600 Rand (1 US $\$=2.7$ Rand in 1991), the median number
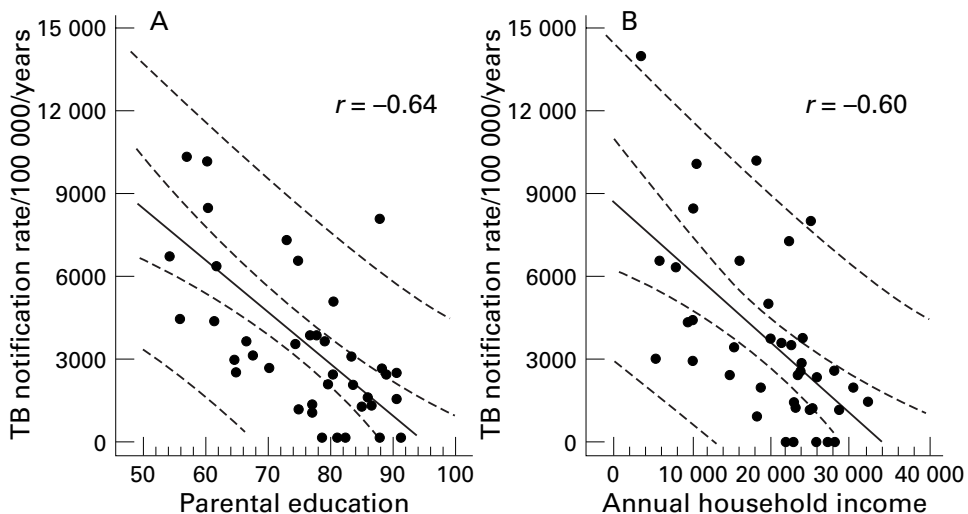

Figure 3 Correlation between TB case notification rate/100 000/year in children 0-5 years old and: $(A)$ maternal education (expressed as percentage of female adults who completed primary education); and (B) average annual household income (expressed in Rand; 1 US\$ $=2.7$ Rand in 1991). of years adults ( $>15$ years old) had attended school was eight years, and the mean level of crowding was 1.03 adults per room. The area with the highest TB case notification rate in children $0-5$ years old (14 062/100 000 children aged $0-5$ years) had a high level of crowding (1.49 adults per room), a low mean annual household income (R4450), and a low parental education level (six years of schooling).

\section{Discussion}

EPIDEMIOLOGY OF TB

A striking result of this study is the extremely high TB case notification rate of 3588/ $100000 /$ year in young children $0-5$ years old. This is 3.5 times higher than the case notification rate in adults. Although these are notification rates with the inevitable problem of inaccuracies, we believe that these rates can be useful as an approximation of incidence. An exact picture of the incidence of childhood TB is difficult because of the difficulties in the diagnosis of childhood TB.

Another remarkable finding of this study is the fact that childhood cases make up $46 \%$ of all notified TB cases. This percentage is falsely high because in our study all duplicate notifications and retreatment cases were eliminated and only the number of new cases were used to calculate case load. Retreatment cases, which are as infectious as new cases, were ignored even though they contribute to the epidemic and the total case load. In this area, retreatment cases make up $30 \%$ of all adult cases and therefore if the total case load includes all notified cases, childhood cases make up $39 \%$ of the total case load.

Young children under the age of 5 years are a vulnerable group and can be seen as the virgin population group with a high risk of infection and disease. Because every TB case in a young child is the consequence of a recent infection, this high childhood case load is an indication of continuing transmission of the disease in the community and of partial failure 
of the TB control programme. ${ }^{1}$ Furthermore, the infected and diseased children, if not correctly treated, form a pool from which future adult cases can arise. It is widely believed that in the developed world, apart from HIV driven epidemics, reactivation is the main cause of adult disease, while in the developing world recent infection is a more important factor. DNA fingerprinting studies in the developing world (including the area in this study) have shown that many of the strains of $M$ tuberculosis are unique representing reactivation disease, while less than half of the strains appear in clusters indicating ongoing recent transmission of disease. ${ }^{12}{ }^{13}$ As much as $50 \%$ of adult TB worldwide may thus arise from the pool of inadequately treated infected and diseased individuals. The importance of children in the epidemiology of TB should therefore not be underestimated.

Socioeconomic conditions have traditionally been cited as risk factors for developing TB. ${ }^{14}$ Several studies have shown a correlation between the rate of infection in households, crowding, and poverty (US), ${ }^{15}$ between TB case notification rate, income, and socioeconomic characteristics (Canada), ${ }^{16}$ and between prevalence of infection, social, and economic environment (Panama). ${ }^{17}$ The case notification rate of childhood TB has been reported to be related to crowding (US), ${ }^{18}{ }^{19}$ income (US), ${ }^{19} 20$ rate of unemployment (US), ${ }^{20}$ infant mortality (US), ${ }^{19}$ and social deprivation (UK). ${ }^{21}$ That all three factors studied by us (crowding, economic status, and parental education) were significantly correlated with the case notification rate of TB in children was thus expected. The correlation between maternal literacy and childhood morbidity and mortality is well known. This study confirms this correlation for childhood TB. The mechanism through which socioeconomic disadvantages influence health is complex as all socioeconomic factors are interrelated. Education is important as it determines the economic status of a person (and thus the ability to afford healthy environments) and their understanding of the disease. The more educated the parent is, the more likely he or she is to seek help when they experience symptoms, potentially resulting in a shorter exposure time of the child to the infectious parent. With a higher education level, the parents are also more likely to understand the importance of screening their children for possible infection with $M$ tuberculosis so that prophylaxis can be given before disease occurs.

CHILDHOOD TB: DIFFERENCES BETWEEN THE DEVELOPING AND DEVELOPED WORLD

The results of our study in a developing country with a high $\mathrm{TB}$ case notification rate illustrates major differences in the epidemiology of childhood TB when compared to studies recently reported from developed countries.

Firstly, studies in the Bronx, New York ${ }^{18}$ showed a significant correlation between the percentage of people living in crowded conditions (more than one person per room) and childhood TB. When this definition of crowding was used in our study, $100 \%$ of people live in crowded conditions and therefore no correlation calculations could be made. When the definition was changed and crowding calculated as adult persons per room, a weak correlation between crowding and TB case notification rate in children was found. This might be because there is a "saturation level" for the influence of crowding and once a certain level of crowding is reached, a further increase does not necessarily increase the risk for transmission of $M$ tuberculosis. Another possible explanation is that in an area with a TB epidemic, the spread of TB is dependent on conditions in the whole community and not only on crowding within the household.

A second remarkable difference is the percentage of childhood cases of the total case load. Percentages for the developed world vary between $2 \%$ and $7 \%,{ }^{11}{ }^{22-24}$ much lower than the $15 \%$ estimation for the developing world in $1990^{25}$ and the $39 \%$ in our study population. This could be explained by a difference in the population structure, with a higher percentage of young children in the developed world (33\% of the population in the studied communities are children $0-14$ years old). A higher percentage of children in the population results in a higher number of children per household (average of three in our study population), and thus a higher chance of an infectious parent infecting at least one of the children. In addition transmission of $M$ tuberculosis from an infectious adult to a child and subsequent development of disease may be enhanced by a combination of the high case notification rate of TB and poor socioeconomic circumstances.

A third difference is the fact that the percentage of older children (5-14 years old) with $\mathrm{TB}$ in this study $(21 \%)$ is significantly lower when compared to $37 \%$ of children in the study from Alabama. ${ }^{11}$ The difference is even larger when one considers only the children in the age group $10-14$ years $\left(3.9 \%\right.$ v $\left.21 \%{ }^{11}\right)$, which illustrates the low TB case notification rate in the "favoured age" group of 6-14 years. ${ }^{26}{ }^{27} \mathrm{~A}$ reason for these differences might be that the contact screening in developed countries is not limited to the age group of $0-5$ years like in our study but extends to all children.

A last difference is the fact that the percentage of extrapulmonary TB in children in the developed world is larger $\left(17.5 \%{ }^{11} v 2 \%\right)$. It is known that as a TB epidemic is overcome, the case notification rate of pulmonary $\mathrm{TB}$ decreases faster compared to the case notification rate of extrapulmonary $\mathrm{TB} .{ }^{27}$ The low percentage of extrapulmonary $\mathrm{TB}$ in our study can further be explained by the fact that more than $97 \%$ of children have received BCG vaccination, which is known to decrease the percentage of extrapulmonary disease. ${ }^{28}$

\section{IMPLICATIONS FOR TB CONTROL PROGRAMMES}

Since the source of infection in every child with $\mathrm{TB}$ is an infectious adult, treating smear positive infectious adults is the priority as this is the key to interrupt transmission of TB. This is best achieved by following WHO guidelines until the goal of $85 \%$ cure of all new smear 
positive cases and $60-85 \%$ case finding is reached. ${ }^{29}$ Active contact tracing and treatment of (smear negative) children only enters at a later stage in the control programme because of its low cost effectiveness. ${ }^{25}$ This is in contrast to the guidelines of the International Union Against Tuberculosis and Lung Disease ${ }^{30}$ and the American Academy of Pediatrics ${ }^{31}$ where active contact tracing, chemoprophylaxis of asymptomatic children, and treatment of symptomatic children is an integral part of the control programme. As children in this community of a developing country constitute a higher percentage of total cases as compared to communities in the developed world $(30 \% v$ $2-7 \%$ ), and knowing that these young children, if not treated, are the reservoir for the adult cases of TB in the next decades, it might be wise to spend part of the budget of the control programme on active contact tracing of children as suggested by the International Union Against Tuberculosis and Lung Disease ${ }^{30}$ and the American Academy of Pediatrics. ${ }^{31}$ As $80 \%$ of children with active TB are aged $0-5$ years, costs can be limited by confining the extent of active contact tracing to that age group. This strategy not only creates the opportunity to treat asymptomatic cases of primary $\mathrm{TB}$ and prevent serious complications of TB disease, but would also enable chemoprophylaxis to be offered to recently infected children. The resources spent on this strategy will not have an immediate effect on the epidemiology of TB in terms of a decrease in the case notification rate of adult disease, but it may help reduce the load of infectious cases in the future. That the impact in the future could be substantial is demonstrated by the fact that DNA fingerprinting analysis suggests that more than half of adult culture proven cases are caused by reactivation of a previous infection, most likely acquired during childhood. ${ }^{12}$ It is therefore encouraging that active contact tracing of children up to 5 years old has become part of the new South African national tuberculosis programme (implemented in 1996), even before the WHO goal of a cure rate and case finding rate of $85 \%$ has been achieved.

A TB epidemic cannot be controlled by treating only the active cases with antituberculous drugs. In addition, even in low income countries, all national tuberculosis programmes should give attention to chemoprophylaxis of infected children. Apart from the political commitment needed for the implementation of a well managed national TB programme, we believe that to eliminate TB, political commitment is also needed to improve the housing, education, and socioeconomic status of the population.

We thank the D Collen Research Foundation (Leuven, Belgium) and Glaxo Wellcome Action TB International Research Initiative for financial support, Dr M Tatley for the notification data, and $\mathrm{M}$ van der Merwe and A Coetzer for secretarial assistance.
1 Starke JR, Jacobs RF, Jereb J. Resurgence of tuberculosis in children. F Pediatr 1992;120:839-55.

2 Bloch AB, Snider DE. How much tuberculosis in children must we accept? Am f Public Health 1985;76:14-15.

3 Starke JR. Tuberculosis in children. In: Reichman LB, Hershfield ES, eds. Tuberculosis: a comprehensive international approach. New York: Marcel Dekker Inc, 1993:329-67.

4 Kochi A. The global tuberculosis situation and the new control strategy of the World Health Organization. Tubercle 1991;72:1-6.

5 Hershfield E. Tuberculosis in children: guidelines for diagnosis, prevention and management (a statement of the scientific committees of the IUATLD). Bulletin of the International Union Against Tuberculosis and Lung Disease 1991;66:61-7.

6 Miller FJW, Seal RME, Taylor MD. Tuberculosis in children. Boston: Little, Brown \& Co, 1963.

7 Osborne CM. The challenge of diagnosing childhood tuberculosis in a developing country. Arch Dis Child 1995; 72:369-74

8 Beyers N, Gie RP, Zietsman L, et al. The use of a geographical information system (GIS) to evaluate the distribution of tuberculosis in a high-case notification rate community. $S$ Afr Med $\mathcal{F}$ 1996;86:41-4

9 Department of National Health and Population Development. Second national survey of women attending
antenatal clinics, South Africa, October/November 1991. Epidemiological Comments 1992;19:80-89.

10 Davies PDO, Darbyshire J, Nunn AJ, et al. Ambiguities and inaccuracies in the notification system for tuberculosis in England and Wales. Community Medicine 1981;3:108-18.

11 Kimerling ME, Vaughn ES, Dunlap NE. Childhood tuberculosis in Alabama: epidemiology of disease and indicators of program effectiveness, 1983 to 1993. Pediatr Infect Dis 7 of program effectiven

12 Warren R, Hauman J, Beyers N, et al. Unexpectedly high train diversity of Mycobacterium tuberculosis in a high-incidence community. S Afr Med f 1996;86:45-9.

13 Pineda-Garcia L, Ferrera A, Hoffner SE. DNA fingerprinting of Mycobacterium tuberculosis strains from patients with pulmonary tuberculosis in Honduras. $\mathcal{F}$ Clin Microbiol 1997;35:2393-7.

14 Dubos R, Dubos J. The white plague: tuberculosis, man, and society. New Brunswick, New Jersey: Rutgers University Press, 1987.

15 Chapman JS, Dyerley MD. Social and other factors in intrafamilial transmission of tuberculosis. Am Rev Respir Dis 1964;90:48-60.

16 Enarson DA, Wang JS, Dirks JM. The incidence of active tuberculosis in a large urban area. Am $\mathcal{f}$ Epidemiol 1989;129:1268-76.

17 Young RV, Pritham H. Prevalence of tuberculin sensitivity in the Panama Canal zone. Diseases of the Chest 1963;43:17275 .

18 Drucker E, Alcabes P, Bosworth W, Sckell B. Childhood tuberculosis in the Bronx, New York. Lancet 1994;343: 1482-5

19 Reinhard C, Paul WS, McAuley JB. Epidemiology of pediatric tuberculosis in Chicago, 1974 to 1994: a continuing public health problem. Am F Med Sci 1997;313:336-40.

20 Chaulk CP, Khoo L, Matuszak DL, Israel E. Case characteristics and trends in pediatric tuberculosis, Maryland, 1986-1993. Public Health Reports 1997;112:146-52.

21 Goldman JM, Teale C, Cundall DB, Pearson SB. Childhood tuberculosis in Leeds, 1982 to 1990: social and ethnic factors and the role of the contact clinic in diagnosis. Thorax 1994;49:184-5.

22 Snider DE, Rieder HL, Combs D, Bloch AB, Hayden CH, Smith MHD. Tuberculosis in children. Pediatr Infect Dis $\mathcal{f}$ 1988;7:271-8.

23 Starke JR. Childhood tuberculosis during the 1990s. Pediatr Review 1992;13:343-53.

24 Ussery XT, Valway SE, McKenna M, Cauthen GM, McCray E, Onorato IM. Epidemiology of tuberculosis among children in the United States: 1985 to 1994. Pediatr Infect Dis f 1996;15:697-704

25 Murray CJL, Stylbo K, Rouillon A. Tuberculosis in developing countries: burden, intervention and cost. Bulletin of the International Union Against Tuberculosis and Lung Disease 1990;65:6-24

26 Starke JR. Modern approach to the diagnosis and treatment of tuberculosis in children. Pediatr Clin North Am 1988;35: 441-65.

27 Comstock GW. Epidemiology of tuberculosis. Am Rev Respir Dis 1982;125:8-15.

28 Fine PEM. Variation in protection by BCG: implications of and for heterologous immunity. Lancet 1995;346:1339-45.

29 World Health Organization. Treatment of tuberculosis: guidelines for national tuberculosis programmes, 2nd ed. Geneva: WHO, 1997.

30 International Union Against Tuberculosis and Lung Disease. Tuberculosis guide for low income countries, 4th ed. Paris: IUATLD, 1996

31 American Academy of Pediatrics Committee on Infectious Diseases. Screening for tuberculosis in infants and children. Pediatrics 1994;93:131-4. 